\title{
Inhibition of the Invasion of Human Glioblastoma U87 Cell Line by Ruxolitinib: A Molecular Player of miR-17 and miR-20a Regulating JAK/STAT Pathway
}

\author{
Emre DELEN ${ }^{1}$, Oguzhan DOGANLAR ${ }^{2}$, Zeynep Banu DOGANLAR ${ }^{2}$, Ozlem DELEN ${ }^{3}$ \\ ${ }^{1}$ Trakya University School of Medicine, Department of Neurosurgery, Edirne, Turkey \\ ${ }^{2}$ Trakya University School of Medicine, Department of Medical Biology, Edirne, Turkey \\ ${ }^{3}$ Trakya University School of Medicine, Department of Histology and Embryology, Edirne, Turkey
}

This study has been presented as an oral presentation at the $33^{\text {rd }}$ Scientific Congress of Turkish Neurosurgical Society between 11 and 14 April 2019 at Antalya, Turkey.

Corresponding author: Emre DELEN emredelen1979@yahoo.com

\section{ABSTRACT}

AIM: To determine the interaction between ruxolitinib, JAK/STAT signalling, and two angio-microRNAs (miRs) to expose potential target molecules in the inhibition of glioblastoma invasion.

MATERIAL and METHODS: The invasion properties of glioblastoma were analyzed using a cancer cell spheroid invasion assay. Following treatment of $195 \mathrm{nM}$ ruxolitinib, the relative expression levels of miR-17 and miR-20a and genes of IL-6/JAK/STAT3 receptor signaling belonging to the JAK/STAT pathway were measured by qRT-PCR in treated and untreated three-dimensional tumor spheres of U87 cells.

RESULTS: Our results indicated that a therapeutic dose of ruxolitinib (195 nM) significantly increased miR-17 and miR-20a expression. Ruxolitinib treatment resulted in the production of IL-6 and active formation of IL-6 receptor complex for the subsequent activation of the IL-6R/JAK2/STAT3 axis. However, ruxolitinib treatment significantly decreased the expression of JAK2 and PI3K. Pearson correlation analyses revealed a strong negative correlation of miR-17 with JAK2, STAT3, and PI3K expressions, and also miR-20a has a negative correlation with expression levels of JAK2 and PI3K. The only positive correlation was found to be between miR-20a and IL-6, gp130 expressions.

CONCLUSION: The specific JAK2 inhibitor ruxolitinib plays an important role in glioblastoma angiogenesis biology via inhibiting IL-6 receptor-dependent JAK/STAT signaling. Additionally, both miR-17a-3p and miR-20a overexpression induced by ruxolitinib treatment may be playing a major role in downregulated JAK2, STAT3, and PI3K proteins. Our results suggest that miR-17-3p and miR-20a-5p may serve as new therapeutic targets for the treatment of glioblastoma.

KEYWORDS: Angiogenesis inhibitors, Drug effects, Glioblastoma, Invasion, Ruxolitinib, JAK/STAT signaling pathway, microRNAs, Molecular targeted therapy

\section{INTRODUCTION}

G lioblastoma multiforme (GBM), also known as grade IV glioma, is the most common type of adult primary brain tumor, and is currently among the incurable forms of cancer. Angiogenesis and invasion are common events that limit treatment of GBM $(33,34,38,39)$. Therefore, understanding the mechanisms underlying and inhibition of these events may be help to inform optimal therapeutic strategies to treaty GBM. 
Interleukin 6 (IL-6) interacts with gp130 and IL-6R to form receptor complex which in turn leads to downstream activation of the Janus kinase/signal transducer and activator of transcription 3 (JAK/STAT3) signaling pathway. Previous studies have shown that the IL-6/JAK/STAT3 signaling pathway is strongly associated with glioblastoma including cell invasion and angiogenesis $(26,30)$. Thus, the inhibition of this pathway may be critical to the prevention of glioblastoma invasion.

Previous studies have reported that two JAK inhibitors, AG490 and WP1066, efficiently suppressed glioblastoma invasion $(19,44)$. Ruxolitinib, an inhibitor of JAK1 and JAK2, is approved by U.S. Food and Drug Administration (FDA) for polycythemia vera and primary myelofibrosis (13). At present, a clinical trial of ruxolitinib in patients with grade III glioma and glioblastoma is ongoing (9).

Non-coding regions of the human genome produce small ribonucleic acid (RNA) molecules of 18-25 nucleotides that are termed microRNAs (miRs). miRs are small players with a pivotal role in the development and progression of gliomas due to their high levels of activation in the post-transcriptional regulation of protein expression. The term angio-miR was coined to identify specific classes of miRs that are involved in angiogenesis, and they were shown to play a potential role in the tumorigenesis of glioblastoma (12). In this regard, miRs have received a great deal of attention as potential therapeutic targets for the treatment of glioblastoma (2).

The aims of this study were to determine the potential role of the ruxolitinib on IL-6/JAK/STAT3 axis of JAK/STAT pathway in glioblastoma invasion, and to investigate the regulatory effect of ruxolitinib induced miR-17 and miR-20a in the glioblastoma tumor spheroid model of the U87 cell line.

\section{MATERIAL and METHODS}

\section{Drugs and Chemicals}

Ruxolitinib (CAS 941678-49-5) was purchased from Santa Cruz Biotechnology (Santa Cruz, CA). The PureLink ${ }^{\circledast}$ RNA Mini Kit, mirVana ${ }^{\mathrm{TM}}$ miRNA Isolation Kit, High Capacity cDNA Reverse Transcription Kit, TaqMan ${ }^{\mathrm{TM}}$ Advanced miR cDNA Synthesis Kit, Power SYBR ${ }^{\circledR}$ Green Master Mix, TaqMan ${ }^{\mathrm{TM}}$ Gene Expression Master Mix, and molecular grade water were obtained from Thermo Fisher Scientific.

\section{Cell Culture, Generation of Spheroids And Treatments}

The human glioblastoma cell line U87-MG (American Type Culture Collection $[\text { ATCC }]^{\circledR}$ HTB-14 ${ }^{\mathrm{TM}}$ ) was cultured in ATCCformulated Eagle-s Minimum Essential Medium (Catalog No. $30-2003), 10 \%$ fetal calf serum (Gibco Life Technologies), $2 \mathrm{mM}$ glutamine (Gibco-Life Technologies), and 1\% final concentration of penicillin/streptomycin (Invitrogen, Life Technologies). Cells were kept in a humidified incubator at $37^{\circ} \mathrm{C}$ and $5 \% \mathrm{CO}_{2}$ during the entire study. Tumor spheroids were generated according to Berens et al. (1). For the treatments, $40 \mu \mathrm{l}$ collected spheroids in medium, $100 \mu \mathrm{L}$ Matrigel matrix (Corning, USA), and $100 \mu \mathrm{L}$ collagen type I (Sigma Aldrich, USA), mixed in a cold Eppendorph tube, and $40 \mu \mathrm{l}$ mixture were plated on six wells of 24-well plates previously coated with Matrigel. The plate was placed into a $37^{\circ} \mathrm{C} 5 \% \mathrm{CO} 2$ incubator to polymerize the three-dimensional culture. Next, $1 \mathrm{~mL}$ of cell culture media was gently added to each well. After 24 hours, the tumor spheroids were treated with 195 $\mathrm{nM}$ ruxolitinib for 48 hours. Six replicates were used for each treatment, and duplicate experiments were performed.

\section{Evaluation of Invasive Activity}

After treatment, cell invasion was recorded for 48 hours using an inverted phase contrast light microscope at 20x magnification on a Zeiss Axio Vert.A1 with digital camera. Two parameters were used in the calculation of invasion capacity: 1) the longest invasive distance emanating from the spheroid, 2) total area invaded by cells leaving the spheroid, as suggested by according to Del Duca et al. (11). Images were analyzed using Image $\mathrm{J}$ software (35).

\section{RNA Isolation, Reverse Transcription and qRT-PCR Assay}

Total RNA was isolated from single spheroids of each group (five replicates) using an RNA purification kit (Thermo Fisher Scientific, USA). The cDNAs were synthesized with a HighCapacity cDNA Reverse Transcription Kit (Thermo Fisher Scientific, USA). MiRs were isolated from the single spheroids of each group (five replicates) using a mirVana ${ }^{\mathrm{TM}}$ miRNA Isolation Kit, and cDNAs were synthesized with an Advanced miRNA cDNA Synthesis Kit, in accordance with kit protocols. Quantitative real time polymerase chain reaction (qRT-PCR) analysis was performed on a QuantStudio 5 Real-Time PCR system (Life Technologies, USA) using the TaqMan ${ }^{\text {TM }}$ Gene Expression Master Mix (Life Technologies, USA). The thermal cycling condition and oligonucleotide primer IDs are shown in Table I. Relative expression levels were calculated using the comparative cycle threshold $(2-\Delta \Delta \mathrm{Ct})$ method, and the ribosomal RNA $18 \mathrm{~S}$ for mRNA and U47 for miR were used as internal controls.

\section{Statistical Analysis}

The differences in the invasion rate, tumor volume, and the relative fold change in gene and miR expression between control and experimental groups were determined using independent sample t-tests. Pearson correlation coefficient were used for correlation analysis. Both statistical analyses were performed using SPSS 20.0 software at a significance level of $p \leq 0.05$.

\section{RESULTS}

The effect of the JAK2 inhibitor ruxolitinib on tumor cell growth and invasion was studied in a three-dimensional culture system. Tumor spheroids of U87-MG cell were treated with $195 \mathrm{nM}$ of ruxolitinib, and the effects on tumor volume and invasion were examined (Figure 1A, B). Although ruxolitinib slightly suppressed growth of tumors spheroids, the reduction in tumor volume of $21.9 \%$ was not statistically significant $(p=0.061)$. However, ruxolitinib significantly inhibited tumor invasion, which served as a biomarker of metastatic potential, via a $95.2 \%$ reduction in invasion area in the three-dimensional culture system of the U87 cell line $(p<0.0001)$. 
Delen E. et al: Inhibition of Glioblastoma Invasion by Ruxolitinib

Table I: Genes, miRNAs, Taq-Man Assay ID and PCR Conditions bb vn elong to qRT-PCR Assay

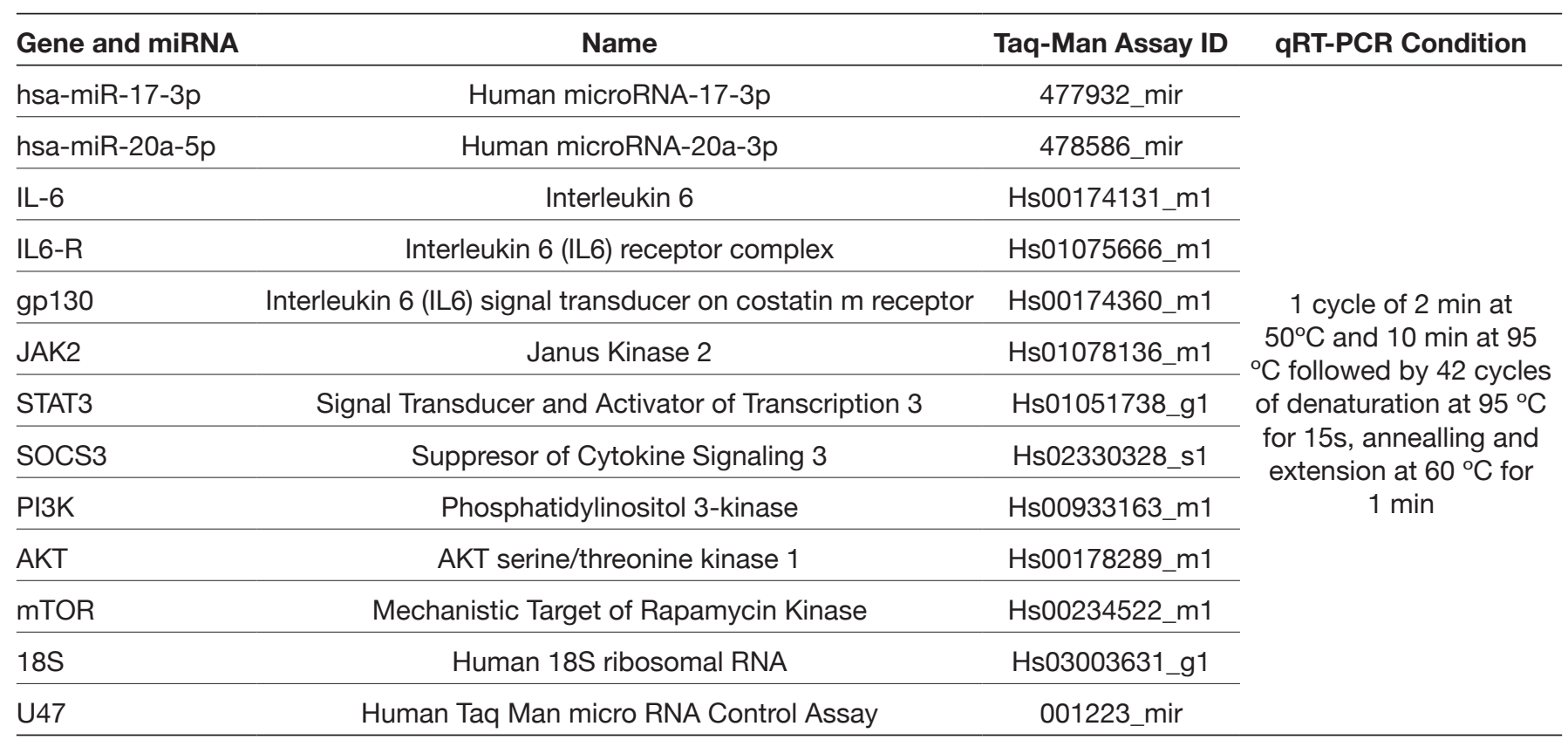

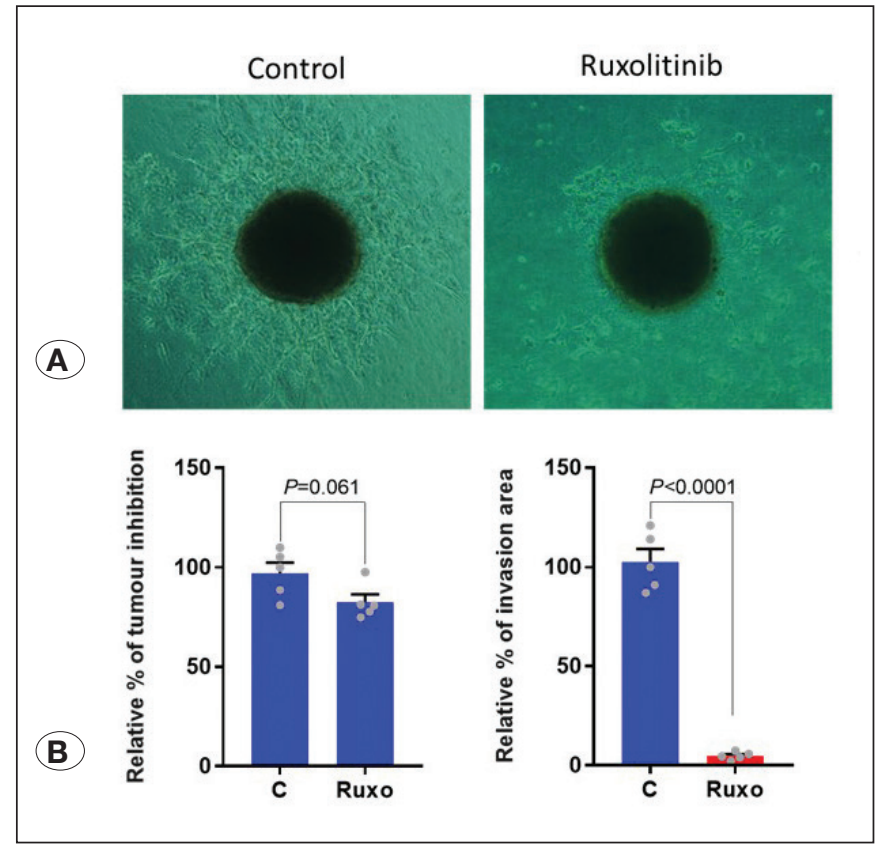

Figure 1: A) Tumour volume and invasion rate of 195nM ruxolitinib treated and untreated (control) U87 spheroids. B) Relative \% of tumour inhibition and relative \% of invasion area in control and experimental groups at 48 hours. Values expressed as mean $\pm \mathrm{SE}$, $\mathrm{n}=5$. Red colour indicates significantly different values compared to control groups (independent-samples t-test: $p \leq 0.05$ ).

To further examine the mechanism that accounts for the inhibition of growth and invasion by ruxolitinib, qRT-PCR in spheroids treated with $195 \mathrm{nM}$ ruxolitinib for 48 hours was performed for miR-17-3p and miR-20a-5p and gene expression of the IL-6 receptor-dependent JAK/STAT pathway. The qRT-
PCR assay revealed a significant upregulation in the relative expression level of both angio-miRs in 195 nM ruxolitinibtreated tumour spheroids compared to the untreated control group (miR-17-3p = 2.75 \pm 0.6-fold, $p=0.023$ and miR-20a-5p $=9.33 \pm 1.85$-fold, $p=0.0011$ ) (Figure 2).

To investigate the effects of ruxolitinib on IL-6/JAK/STAT signaling, the mRNA levels of two key IL-6 receptors, gp130 and IL-6R with IL-6 cytokine genes, were determined. Whereas ruxolitinib treatment caused significant upregulation of IL-6 and gp130, there was no significant change in IL$6 \mathrm{R}$ level (Figure 3). This finding indicated that IL-6 receptor signaling occurred in ruxolitinib-treated glioblastoma tumor spheroids. To check if whether IL-6 receptor-dependent JAK/ STAT signal was generated, we examined the gene expression levels in the JAK/STAT and PI3K/AKT/mTOR axis activated by the initiation signal. Ruxolitinib caused a significant decrease in JAK2, the main initial factor of IL-6/JAK/STAT axis, and in SOCs3 gene expression levels compared to their respective controls; additionally, $\mathrm{PI} 3 \mathrm{~K}$, the primary initial protein of the IL-6/PI3K/AKT/mTOR axis, was significantly suppressed by drug treatments at 48 hours in U87 tumor spheroids (Figure 3). In order to determine the interaction between miRs and the JAK/STAT signalling genes, Pearson correlation analyses were performed on the relative gene expression level of these molecular markers. This analyse revealed a strong negative correlation of miR-17 with JAK2, STAT3, and PI3K expressions, while miR-20a has a negative correlation with expression levels of JAK2 and PI3K. The only positive correlation was found to be between miR-20a and IL-6, gp130 expressions (Table II).

\section{DISCUSSION}

Ruxolitinib, an orally administered inhibitor of JAK1 and JAK2, 


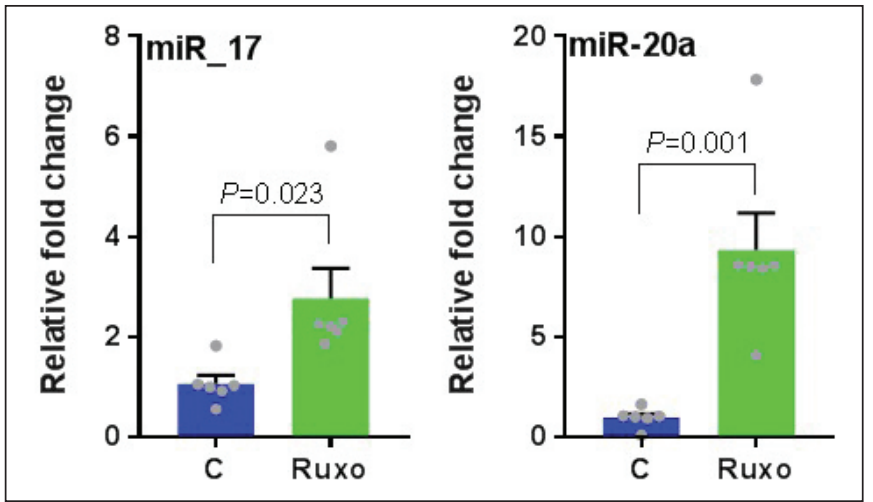

Figure 2: Relative fold change determined by quantitative realtime PCR (qRT-PCR) analysis of miR-17-3p and miR-20a-5p in control and $195 \mathrm{nM}$ ruxolitinib exposed U87 tumour spheroid. All data were normalized with U47 expression and given as relative to control. Values expressed as mean $\pm S E, n=6$. Green colour indicates significantly different values compared to control groups (independent-samples t-test: $\mathrm{p} \leq 0.05$ ). is used clinically in patients with myeloproliferative neoplasms such as polycythemia vera and primary myelofibrosis $(13,27)$. Several previous studies have demonstrated an anti-tumor effect of ruxolitinib in, human ovarian cancer, non-small-cell lung cancer models, and pancreatic cancer via JAK/STAT pathway inhibition $(18,32,43)$. Here, our study revealed that ruxolitinib strongly inhibits the invasion of human glioblastoma U87 cells via the IL-6/JAK/STAT3 signaling pathway. In addition, we found that a strong negative correlation existed between miR-17 and JAK2, and STAT3 and PI3K, and a significant negative correlation existed between miR-20a and JAK2, PI3K expressions.

The formation of new blood vessels by angiogenesis plays a key role in glioblastoma invasion involving various physiological processes, including the IL-6/JAK-STAT3 signaling pathway. The median survival in patients with GBM is poor because of local recurrence due to invasion of glioma cells into normal brain tissue $(4,17,22,33)$. Moreover, this fact is one of the reason for the non-curative nature of surgical
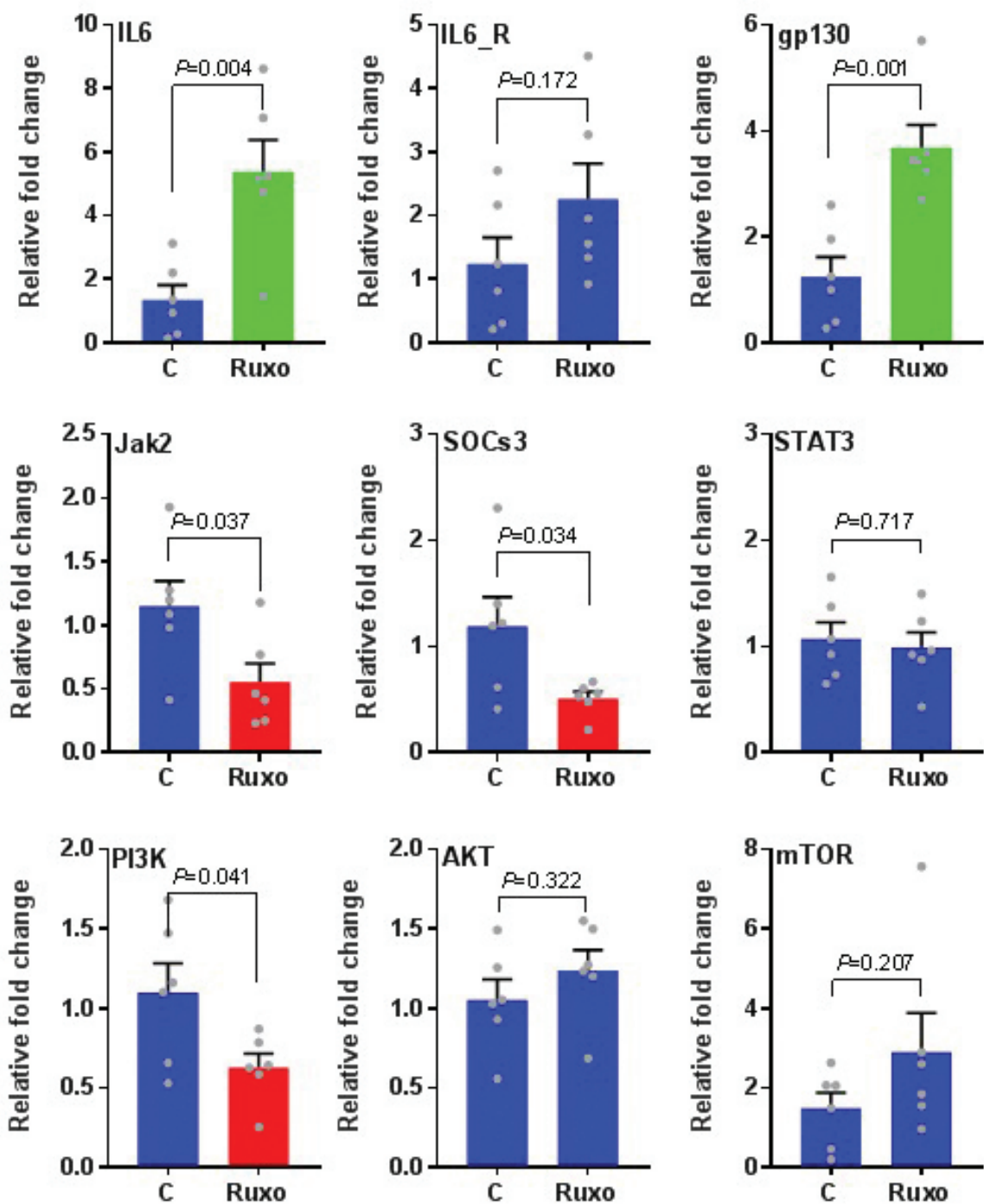
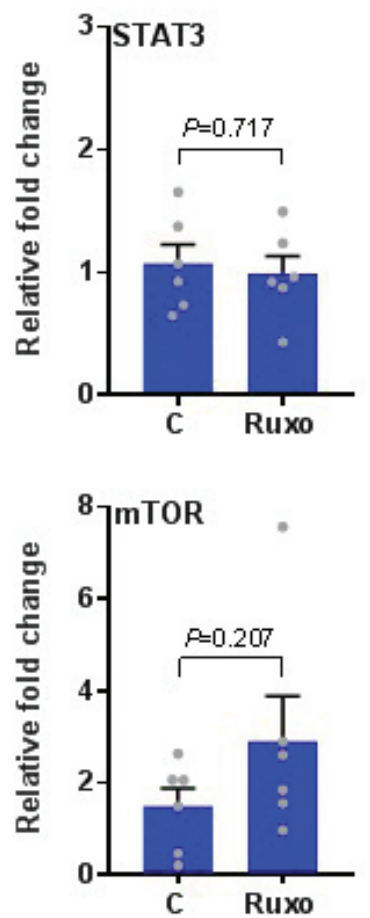

Figure 3: Relative fold change determined by quantitative real-time PCR (qRT-PCR) analysis of Jak/StatIL6 receptor signalling miR-17-3p and miR-20a-5p in control and 195 nM ruxolitinib exposed U87 tumour spheroid. All data were normalized with GAPDH expression and given as relative to control. Values expressed as mean $\pm \mathrm{SE}, \mathrm{n}=6$. Green and red colours indicate significantly different values compared to control groups (independent-samples t-test: $\mathrm{p} \leq 0.05$ ). 
Delen E. et al: Inhibition of Glioblastoma Invasion by Ruxolitinib

Table II: Correlation Between Relative Expression Level of Angio-miRs and JAK/STAT-IL 6 Receptor Signalling Genes

\begin{tabular}{lcccc}
\hline & \multicolumn{2}{c}{ miR-17 } & \multicolumn{2}{c}{ miR-20a } \\
\hline Genes & Pearson Correlation & Sig. (2-tailed) & Pearson Correlation & Sig. (2-tailed) \\
\hline Jak2 &,,$- 696^{*}$ &, 012 &,$- 725^{* *}$ &, 008 \\
\hline Stat3 &,$- 604^{*}$ &, 038 &,- 489 &, 106 \\
\hline Pl3K &,$- 738^{* *}$ &, 006 &,$- 712^{* *}$ &, 009 \\
\hline IL6 &, 453 &, 139 &, $684^{*}$ &, 014 \\
\hline IL6R &,- 118 &, 714 &, 013 &, 969 \\
\hline gp130 &, 490 &, 106 &, $660^{*}$ &, 019 \\
\hline Socs3 &,- 446 &, 146 &,- 498 &, 099 \\
\hline Akt &, 262 &, 410 &, 395 &, 204 \\
\hline mTOR &, 216 &, 500 &, 291 &, 359 \\
\hline
\end{tabular}

${ }^{* *}$ Correlation is significant at the 0.01 level (2-tailed).

${ }^{*}$ Correlation is significant at the 0.05 level (2-tailed).

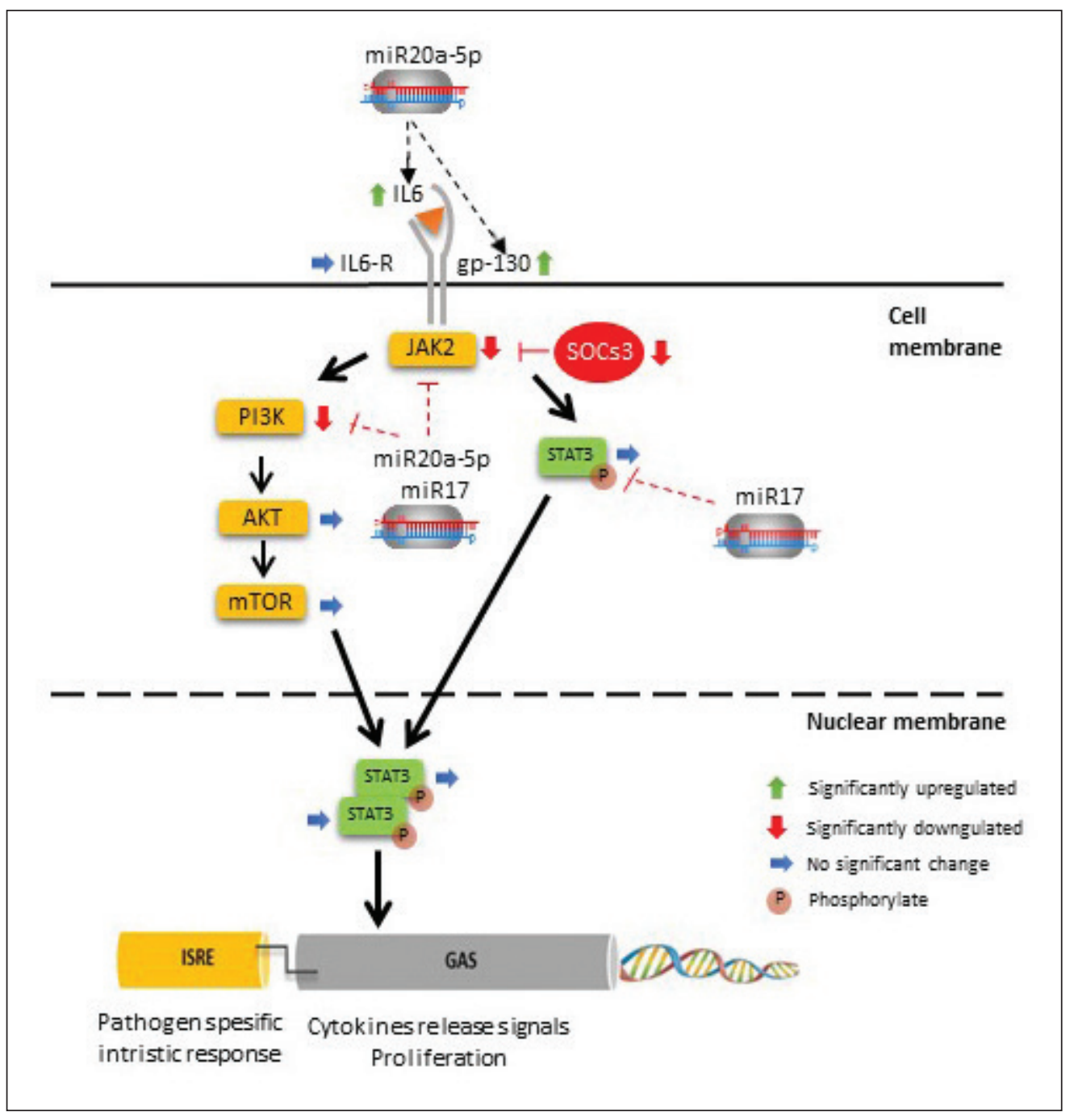

Figure 4: Jak/Stat-IL 6 receptor signalling pathway and gene and miRNAs expression status in ruxolitinib treated tumour spheroids of U87 cells. 
Delen E. et al: Inhibition of Glioblastoma Invasion by Ruxolitinib

resection of GBM. Consequently, treatment of peritumoral invasion in glioblastoma might be a possible curative treatment strategy. In this regard, previous studies reported that to prevent glioblastoma invasion for the treatment of GBM $(8,17,23,44)$. Similarly, after treatement with ruxolitinib, the current study clearly demonstrates a $95.2 \%$ reduction in invasion area in the three-dimensional culture system of the U87 cell line, thereby suggesting that this method may hold promise for GBM treatment (Figure 1).

Once activated by IL-6, JAK2 initiates a cascade of signaling events that trigger phosphorylation of STAT3. JAK2 is responsible for both mTOR and STAT3 signaling pathway activations (Figure 4). The mTOR signaling pathway was considered separately in the following paragraphs. IL-6/ JAK2/STAT3 axis is an important molecular pathway strongly associated with a variety of cancer types as well as glioblastoma $(3,15,19)$. Moreover, Liu et al demonstrated that IL-6 promotes glioblastoma invasion through the JAK/ STAT3 pathways (26). Thus, this pathway inhibition, achieved by blocking any molecular players involved in this process, may be a good strategy for treatment of GBM. Tocilizumab, a humanized monoclonal antibody against IL-6R, has an antiproliferative effect on U87-MG glioma cells via this pathway (21). A previous preclinical study showed that low concentrations of pacritinib, an inhibitor of JAK, inhibits the JAK2/STAT3 signaling pathway with minimal effects on other signalling pathways (20). At higher concentrations of pacritinib, phosphorylation of AKT is reduced, which has an effect on the mTOR axis. In the current study, a single dose of ruxolitinib $(195 \mathrm{nM})$ is shown to be adequate for the prevention of invasion via inhibition of JAK/STAT3 signaling pathway. However, the efficacy of different doses of ruxolitinib in the other signaling pathways has not been investigated, which is a limitation of our study. Weissenberger et al. revealed that STAT3 activation increases with the malignancy of the glioma (41). Cardamonin, an inhibitor of STAT3, has been found to suppress proliferation of glioblastoma stem cells (42). Additionally, it was shown that the anti-helminthic drug niclosamide, another STAT3 inhibitor, significantly inhibits cell proliferation in U87-MG cells. As a result, growing evidence has demonstrated that JAK2/STAT3targeted therapy suppresses glioblastoma invasion $(29,44)$. In this study, we clearly demonstrate that a single dose of ruxolitinib suppressed the invasion of glioblastoma via inhibition of the IL-6/JAK2/STAT3 axis.

Clark et al. showed that resveratrol inhibited U-87 cells in filtration via reduced $A K T$ phosphorylation which has a pivotal role on the mTOR pathway (8). The PI3K/AKT/mTOR pathway, which is triggerred by JAK2, plays an important role in many cellular functions such as growth control, apoptosis, and metabolism. Activation of this pathway is associated with poor prognosis of patients with GBM (6). To date, although inhibitors intended to target this pathway have failed to improve the treatment of patients with GBM, previous studies support the hypothesis that this pathway is still currently one of the most attractive therapeutic targets in $\operatorname{GBM}(25,28)$. Speranza et al. showed that BKM-120, a P3IK inhibitor, may have anti-invasive properties in glioblastoma (36). Under hypoxia conditions, it was reported that the mTOR pathway participates in regulating invasion of U87 cell lines (17). In the U251-MG cell lines, oxymatrine, a natural quinolizidine alkaloid extracted with antitumor activities, decreased the expression levels of p-AKT and p-mTOR (10). Inhibition of this pathway, especially the downregulation of PI3K, was observed in the present study (Figure 3). In addition, a negative correlation between miR-17, miR-20a, and PI3K was exhibited (Table II).

MiRs have a pivotal role in cell functions including proliferation, apoptosis, differentiation, migration, angiogenesis, and so on in the tumorigenesis of glioblastoma $(2,31,37,40)$. Additionally, multiple studies have shown that angiomiRs regulate the expression of genes, which have a pivotal role in epigenetic modification, being involved in both angiogenesis and invasion of glioblastoma (2). STAT3 is the most studied stat protein in the JAK/STAT pathway and is regulated by miR-17 cluster family members. The inhibitory effect of miR-17-92 on STAT 3 has been shown to be very important in tumor-like processes, as well as in hypoxia and angiogenesis $(7,14,16)$. MiRs-17, $-20 \mathrm{a}$ and $-106 \mathrm{~b}$ were determined to target STAT3 and MAPK14 directly (5). Additionally, it was shown that the expression of miR-17, an angiomiR, promoted glioblastoma cell invasion (24). In the present study, after treatment of ruxolitinib, while expression of both miR-17 and miR-20a were significantly up-regulated, strong downregulation was observed in JAK2, STAT3 and PI3K expression. In addition, significant correlations among these miRs and genes show gene-miRs interactions at molecular levels and are supportive of our data (Table II). These results suggest that miR-17 and miR-20a may regulate cell invasion of glioblastoma via IL-6/ JAK2/STAT3 axis belonging to the JAK/STAT pathway (Figure 4).

\section{CONCLUSION}

Our findings suggest that JAK/STAT signaling acts as a proangiogenic factor in glioblastoma, and ruxolitinib plays an important role in GBM angiogenesis via both inhibiting IL-6/ JAK2/STAT3 axis and significantly inducing miR-17a-3p and miR-20a expression which that may downregulate JAK2, STAT3, and PI3K. We believe that the combined application of ruxolitinib and miR-17/-20a may serve as an attractive strategy for the therapeutic intervention for glioblastoma in the future.

\section{ACKNOWLEDGMENT}

This study was funded by the Trakya University Scientific Research (TUBAP-2018/155).

\section{REFERENCES}

1. Berens EB, Holy JM, Riegel AT, Wellstein A: A cancer cell spheroid assay to assess invasion in a $3 D$ setting. J Vis Exp 20:1-6, 2015

2. Beyer S, Fleming J, Meng W, Singh R, Haque SJ, Chakravarti A: The role of miRNAs in angiogenesis, invasion and metabolism and their therapeutic implications in gliomas. Cancers (Basel) 9(7).pii: E85, 2017 
3. Brantley EC, Benveniste EN: STAT-3: A molecular hub for signaling pathways in gliomas emily. Mol Cancer Res 6:1-20, 2014

4. Bush NA, Chang SM, Berger MS: Current and future strategies for the treatment of glioma. Neurosurg Rev 40:1-14, 2017

5. Carraro G, El-Hashash A, Guidolin D, Tiozzo C, Turcatel G, Young BM, De Langhe SP, Bellusci S Shi W, Parnigotto PP, Warburton D: miR-17 family of microRNAs controls FGF10mediated embryonic lung epithelial branching morphogenesis. Dev Biol 333:238-250, 2009

6. Chakravarti A, Zhai G, Suzuki Y, Sarkesh S, Black PM, Muzikansky A, Loeffler JS: The prognostic significance of phosphatidylinositol 3-kinase pathway activation in human gliomas. J Clin Oncol 22(10):1926-1933, 2004

7. Chen L, Li C, Zhang R, Gao X, Qu X, Zhao M, Qiao C, Xu J, Li J: MiR-17-92 cluster microRNAs confers tumorigenicity in multiple myeloma. Cancer Lett 309(1):62-70, 2011

8. Clark PA, Bhattacharya S, Elmayan A, Darjatmoko SR, Thuro BA, Yan MB, van Ginkel PR, Polans AS, Kuo JS: Resveratrol targeting of AKT and p53 in glioblastoma and glioblastoma stem-like cells to suppress growth and infiltration. J Neurosurg 126(5):1448-1460, 2017

9. ClinicalTrials.gov [database online]. Bethesda, MD: National Library of Medicine (US). NLM Indentifier: NCT03514069, 2018. Updated June 8, 2018

10. Dai Z, Wang L, Wang X, Zhao B, Zhao W, Bhardwaj SS, Ye J, Yin Z, Zhang J, Zhao S: Oxymatrine induces cell cycle arrest and apoptosis and suppresses the invasion of human glioblastoma cells through the EGFR/PI3K/Akt/mTOR signaling pathway and STAT3. Oncol Rep 40(2):867-876, 2018

11. Del Duca D, Werbowetski T, Del Maestro RF: Spheroid preparation from hanging drops: Characterization of a model of brain tumor invasion. J Neurooncol 67(3):295-303, 2004

12. Di Stefano AB, Massihnia D, Grisafi F, Castiglia M, Toia F, Montesano L, Russo A, Moschella F, Cordova A: Adipose tissue, angiogenesis and angio-MIR under physiological and pathological conditions. Eur J Cell Biol 2018 (Available online)

13. Greenfield G, McPherson S, Mills K, McMullin MF: The ruxolitinib effect: Understanding how molecular pathogenesis and epigenetic dysregulation impact therapeutic efficacy in myeloproliferative neoplasms. J Transl Med 16(1):360, 2018

14. He M, Wang QY, Yin QQ, Tang J, Lu Y, Zhou CX, Duan CW, Hong DL, Tanaka T, Chen GQ, Zhao Q: HIF-1 downregulates miR-17/20a directly targeting p21 and STAT3: A role in myeloid leukemic cell differentiation. Cell Death Differ 20(3):408-418, 2013

15. Hodge DR, Hurt EM, Farrar WL: The role of IL-6 and STAT3 in inflammation and cancer. Eur J Cancer 41(16):2502-2512, 2005

16. Hong L, Lai M, Chen M, Xie C, Liao R, Kang YJ, Xiao C, Hu WY, Han J SP: The miR-17-92 cluster of microRNAs confers tumorigenicity by inhibiting oncogene-induced senescence. Cancer Res 70(21):8547-8557, 2010

17. Huang W, Ding X, Ye H, Wang J, Shao J, Huang T: Hypoxia enhances the migration and invasion of human glioblastoma U87 cells through PI3K/Akt/mTOR/HIF-1a pathway. Neuroreport 29(18):1578-1585, 2018
18. Hurwitz HI, Uppal N, Wagner SA, Bendell JC, Beck JT, Wade SM, Nemunaitis JJ, Stella PJ, Pipas JM, Wainberg ZA, Manges R, Garrett WM, Hunter DS, Clark J, Leopold L, Sandor V, Levy RS: Randomized, double-blind, phase II study of ruxolitinib or placebo in combination with capecitabine in patients with metastatic pancreatic cancer for whom therapy with gemcitabine has failed. J Clin Oncol 33(34):4039-4047, 2015

19. Iwamaru A, Szymanski S, Iwado E, Aoki H, Yokoyama T, Fokt I, Hess K, Conrad C, Madden T, Sawaya R, Kondo S, Priebe W, Kondo Y: A novel inhibitor of the STAT3 pathway induces apoptosis in malignant glioma cells both in vitro and in vivo. Oncogene 26(17):2435-2444, 2007

20. Jensen KV, Cseh O, Aman A, Weiss S, Luchman HA: The JAK2/STAT3 inhibitor pacritinib effectively inhibits patientderived GBM brain tumor initiating cells in vitro and when used in combination with temozolomide increases survival in an orthotopic xenograft model. PLoS One 12(12):1-18, 2017

21. Kudo $M$, Jono $H$, Shinriki $S$, Yano $S$, Nakamura $H$, Makino K, Hide T, Muta D, Ueda M, Ota K, Ando Y, Kuratsu J: Antitumor effect of humanized anti-interleukin-6 receptor antibody (tocilizumab) on glioma cell proliferation. Laboratory investigation. J Neurosurg 111(2): 219-225, 2009

22. Lemée J-M, Clavreul A, Aubry M, Com E, de Tayrac M, Eliat PA, Henry C, Rousseau A, Mosser J, Menei P: Characterizing the peritumoral brain zone in glioblastoma: A multidisciplinary analysis. J Neurooncol 122(1):53-61, 2015

23. Li L, Gondi CS, Dinh DH, Olivero WC, Gujrati M, Rao JS: Transfection with anti-p65 intrabody suppresses invasion and angiogenesis in glioma cells by blocking NF-KB transcriptional activity. Clin Cancer Res 13(7):2178-2190, 2008

24. Li H, Yang BB: Stress response of glioblastoma cells mediated by miR-17-5p targeting PTEN and the passenger strand miR17-3p targeting MDM2. Oncotarget 3(12):1653-1668, 2012

25. Litzlbauer M: Targeting the RTK-PI3K-mTOR axis in malignant glioma: Overcoming resistance. World Electr Veh J 5(3):629634, 2012

26. Liu Q, Li G, Li R, Shen J, He Q, Deng L, Zhang C, Zhang J: IL-6 promotion of glioblastoma cell invasion and angiogenesis in U251 and T98G cell lines. J Neurooncol 100(2):165-176, 2010

27. McKeage K: Ruxolitinib: A review in polycythaemia vera. Drugs 75(15):1773-1781, 2015

28. Mecca C, Giambanco I, Donato R, Arcuri C: Targeting mTOR in glioblastoma: Rationale and preclinical/clinical evidence. Dis Markers 2018:9230479, 2018

29. Mukthavaram R, Ouyang $X$, Saklecha R, Jiang $P$, Nomura N, Pingle SC, Guo F, Makale M, Kesari S: Effect of the JAK2/STAT3 inhibitor SAR317461 on human glioblastoma tumorspheres. J Transl Med 13(1):1-10, 2015

30. Natsume A, Kinjo S, Yuki K, Kato T, Ohno M, Motomura K, Iwami K, Wakabayashi T: Glioma-initiating cells and molecular pathology: Implications for therapy. Brain Tumor Pathol 28(1): 1-12, 2011

31. Pan Y, Liang W, Zhao X, Liu L, Qing Y, Li Y: MiR-548b inhibits the proliferation and invasion of malignant gliomas by targeting metastasis tumor-associated protein-2. Neuroreport 27(17): 1266-1273, 2016 
32. Patel MR, Dash A, Jacobson BA, Ji Y, Baumann D, Ismail K, Kratzke RA: JAK/STAT inhibition with ruxolitinib enhances oncolytic virotherapy in non-small cell lung cancer models. Cancer Gene Ther 2019 (Epub ahead of print)

33. Price SJ, Gillard JH: Imaging biomarkers of brain tumour margin and tumour invasion. Br J Radiol 84(2):159-167, 2011

34. Sayegh ET, Kaur G, Bloch O, Parsa AT: Systematic review of protein biomarkers of invasive behavior in glioblastoma. Mol Neurobiol 49(3):1212-1244, 2014

35. Schneider CA, Rasband WS, Eliceiri KW: NIH image to image $\mathrm{J}$ : 25 years of image analysis. Nat Methods 9(7):671-675, 2012

36. Speranza MC, Nowicki MO, Behera P, Cho CF, Chiocca EA, Lawler SE: BKM-120 (Buparlisib): A phosphatidyl-inositol-3 kinase inhibitor with anti-invasive properties in glioblastoma. Sci Rep 6:1-9, 2016

37. Staszel T, Zapała B, Polus A, Sadakierska-Chudy A, Kieć-Wilk B, Stępień E, Wybrańska I, Chojnacka M, Dembińska-Kieć A: Role of microRNAs in endothelial cell pathophysiology. Pol Arch Med Wewn 121(10):361-367, 2011

38. Tate MC, Aghi MK: Biology of angiogenesis and invasion in glioma. Neurotherapeutics 6(3):447-457, 2009

39. Virga J, Bognár L, Hortobágyi T, Zahuczky G, Csősz É, Kalló G, Tóth J, Hutóczki G, Reményi-Puskár J, Steiner L, Klekner A. Prognostic role of the expression of invasion-related molecules in glioblastoma. J Neurol Surgery Part A Cent Eur Neurosurg 78(1):12-19, 2017
40. Wang Z, Wang B, Shi Y, Xu C, Xiao HL, Ma LN, Xu SL, Yang L, Wang QL, Dang WQ, Cui W, Yu SC, Ping YF, Cui YH, Kung HF, Qian C, Zhang X, Bian XW: Oncogenic miR-20a and miR$106 a$ enhance the invasiveness of human glioma stem cells by directly targeting TIMP-2. Oncogene 34(11):1407-1419, 2015

41. Weissenberger J, Loeffler S, Kappeler A, Kopf M, Lukes A, Afanasieva TA, Aguzzi A, Weis J: IL-6 is required for glioma development in a mouse model. Oncogene 23(19):33083316, 2004

42. Wu N, Liu J, Zhao X, Yan Z, Jiang B, Wang L, Cao S, Shi D, Lin X: Cardamonin induces apoptosis by suppressing STAT3 signaling pathway in glioblastoma stem cells. Tumor Biol 36(12):9667-9676, 2015

43. Yim JH, Jove R, Lu SA, Han ES, Wen W, Wu J, Dellinger TH: Ruxolitinib synergistically enhances the anti-tumor activity of paclitaxel in human ovarian cancer. Oncotarget 9(36):2430424319, 2018

44. Zheng Q, Han L, Dong Y, Tian J, Huang W, Liu Z, Jia X, Jiang T, Zhang J, Li X, Kang C, Ren H: JAK2/STAT3 targeted therapy suppresses tumor invasion via disruption of the EGFRvIlI/ JAK2/STAT3 axis and associated focal adhesion in EGFRvIllexpressing glioblastoma. Neuro Oncol 16(9):1229-1243, 2014 